\title{
Rottlerin inhibits cell growth and invasion via down-regulation of Cdc20 in glioma cells
}

\author{
Lixia Wang ${ }^{1}$, Yingying Hou ${ }^{1}$, Xuyuan Yinn ${ }^{1}$, Jingna Su ${ }^{1}$, Zhe Zhao ${ }^{1}$, Xiantao Ye ${ }^{1}$, Xiuxia \\ Zhou $^{1}$, Li Zhou ${ }^{2}$, Zhiwei Wang ${ }^{1,3}$ \\ ${ }^{1}$ The Cyrus Tang Hematology Center and Collaborative Innovation Center of Hematology, Jiangsu Institute of Hematology, \\ The First Affiliated Hospital, Soochow University, Suzhou, China \\ ${ }^{2}$ Department of Gynecologic Oncosurgery, Jilin Province Cancer Hospital, Changchun, Jilin, China \\ ${ }^{3}$ Department of Pathology, Beth Israel Deaconess Medical Center, Harvard Medical School, USA \\ Correspondence to: Li Zhou, email: libyacynthia@163.com \\ Zhiwei Wang, email: zwang6@bidmc.harvard.edu \\ Keywords: rottlerin, Cdc20, glioma, growth, invasion
}

Received: June 30, $2016 \quad$ Accepted: September 05, $2016 \quad$ Published: September 12, 2016

\section{ABSTRACT}

Rottlerin, isolated from a medicinal plant Mallotus phillippinensis, has been demonstrated to inhibit cellular growth and induce cytoxicity in glioblastoma cell lines through inhibition of calmodulin-dependent protein kinase III. Emerging evidence suggests that rottlerin exerts its antitumor activity as a protein kinase C inhibitor. Although further studies revealed that rottlerin regulated multiple signaling pathways to suppress tumor cell growth, the exact molecular insight on rottlerinmediated tumor inhibition is not fully elucidated. In the current study, we determine the function of rottlerin on glioma cell growth, apoptosis, cell cycle, migration and invasion. We found that rottlerin inhibited cell growth, migration, invasion, but induced apoptosis and cell cycle arrest. Mechanistically, the expression of Cdc20 oncoprotein was measured by the RT-PCR and Western blot analysis in glioma cells treated with rottlerin. We observed that rottlerin significantly inhibited the expression of Cdc20 in glioma cells, implying that Cdc20 could be a novel target of rottlerin. In line with this, over-expression of $\mathrm{Cdc20}$ decreased rottlerin-induced cell growth inhibition and apoptosis, whereas down-regulation of Cdc20 by its shRNA promotes rottlerin-induced anti-tumor activity. Our findings indicted that rottlerin could exert its tumor suppressive function by inhibiting Cdc20 pathway which is constitutively active in glioma cells. Therefore, down-regulation of Cdc20 by rottlerin could be a promising therapeutic strategy for the treatment of glioma.

\section{INTRODUCTION}

Cerebral glioma is the most common brain tumor, which is one of the top ten malignant tumors in human [1]. Among primary malignant brain tumors, 80 percent tumors are malignant gliomas (MGs). Glioblastoma multiforme (GBM) accounts for more than half of MG cases [2]. MGs have been reported to be associated with high morbidity and mortality [3]. Multiple studies have demonstrated that many potential risk factors could contribute to brain tumors, although detailed explanation how these factors are involved in brain tumorigenesis is unclear. The most important factors of brain tumor development include exposure to ionizing radiation, and history of allergy or atopic disease [4]. Surgery is often difficult to remove entire brain tumor and chemotherapeutic drugs cannot pass the blood-brain barrier [5]. Therefore, current combinations of surgical resection, radiation therapy and chemotherapy regimens fail to significantly improve longterm patient survival for these brain cancers [6]. Thus, it is pivotal to discover the new therapeutic agents for the treatment of glioma.

Rottlerin, also called mallotoxin, is isolated from plant Mallotus phillippinensis [7]. Initial studies identified that rottlerin is an inhibitor of PKC $\delta$ (protein kinase $\mathrm{C} \delta$ ) [8]. In recent years, increasing evidence implicated that rottlerin exhibited its anti-tumor activity through inhibition of cell proliferation [9] and migration [10], induction of apoptosis [11] and cell cycle arrest [12] in a variety of human cancer cells. Interestingly, rottlerin 
was discovered to induce autophagy and apoptotic cell death through $\mathrm{PKC} \delta$-independent pathway [13]. $\mathrm{Li}$ et al. also found that rottlerin induced proapoptotic endoplasmic reticulum stress via $\mathrm{PKC} \delta$-independent pathway in human colon cancer cells [14]. Similarly, rottlerin induced apoptosis through upregulation of DR5 (death receptor 5) via $\mathrm{PKC} \delta$-independent pathway in human malignant tumor cells [14]. Mechanistically, rottlerin was reported to inhibit the NF- $\mathrm{KB}$ (nuclear factor kappa B)/cyclin D1 cascade in breast cancer cells [15]. Moreover, Ohno et al. found that rottlerin stimulates apoptosis in pancreatic cancer cells through disrupting the interactions between prosurvival $\mathrm{Bcl}-2$ proteins and proapoptotic $\mathrm{BH} 3$-only proteins [16]. Although multiple studies identified the molecular insight onto rottlerininduced tumor suppression, the underlying mechanisms are still elusive.

Ubiquitination by the UPS (ubiquitin proteasome system) controls cell cycle progression via protein degradation [17]. APC/C (anaphase promoting complex/C) is a multi-subunit E3 ubiquitin ligase, which forms $\mathrm{APC}^{\mathrm{Cdc} 20}$ to exert its biological functions largely through targeting its downstream substrates for ubiquitination and subsequent degradation [18-20]. Emerging evidence has demonstrated that Cdc20 (cell division cycle 20) has an oncogenic function in tumorigenesis [18]. Overexpression of Cdc20 has been identified in a broad spectrum of human cancers and is associated with poor prognosis in various types of cancers [21-23]. For example, overexpression of Cdc20 was observed in glioblastomas, whereas Cdc20 was underexpressed in low-grade gliomas [24]. Furthermore, Cdc20 level was significantly correlated with glioma grade and survival time [25]. Mechanistically, it has been found that $\mathrm{APC} / \mathrm{C}$ (Cdc20) controls the ubiquitin-mediated degradation of p21 in prometaphase [26]. In addition, one study reported that $\mathrm{Cdc} 20$-mediated degradation of conductin regulated Wnt/beta-catenin signaling for maximal activity during G1/S [27]. Moreover, Cdc20 has been identified to be negatively regulated by p53 [28]. These reports indicated that $\mathrm{Cdc} 20$ could be a potential therapeutic target for combating human cancers.

In the current study, we investigated whether Cdc20 plays an important role in regulation of cell growth, apoptosis, cell cycle, migration and invasion in glioma cells. Moreover, we explored whether rottlerin could inhibit the expression of Cdc20 in glioma cells. Furthermore, we determined whether rottlerin exerts its anticancer function via inactivation of Cde20 in glioma cells. We found that rottlerin suppressed cell growth and induced apoptosis and cell cycle arrest in glioma cell lines. We also demonstrated that rottlerin could down-regulate the expression of $\mathrm{Cdc} 20$, leading to anti-tumor activity in glioma cells. Therefore, rottlerin could be a potential efficient agent to inhibit $\mathrm{Cdc} 20$ in glioma.

\section{RESULTS}

\section{Rottlerin inhibited glioma cell proliferation}

Rottlerin has been reported to exhibit anti-proliferation in human cancer cells. To determine whether rottlerin could inhibit the glioma cells growth, MTT assay was performed in U251 and SNB19 glioma cells treated with different concentrations of rottlerin for $48 \mathrm{~h}$ and $72 \mathrm{~h}$. We observed that rottlerin treatment caused cell growth inhibition in the time- and dose- dependent manners in glioma cells (Figure 1A). Our MTT results have clearly demonstrated that rottlerin inhibited cell proliferation in glioma cells.

\section{Rottlerin induced cell apoptosis in glima cells}

Next, we determine whether rottlerin could trigger cell apoptosis in glioma cells, PI-FITC-annexin assay was conducted in U251 and SNB19 glioma cells treated with $2 \mu \mathrm{M}$ and $4 \mu \mathrm{M}$ rottlerin for 48 hours. We found that $2 \mu \mathrm{M}$ and $4 \mu \mathrm{M}$ rottlerin induced cell apoptosis from $4.15 \%$ to $14.01 \%$, to $22.07 \%$, respectively, in U251 cells (Figure 1B). Similarly, $4 \mu \mathrm{M}$ rottlerin treatments led to cell apoptosis from $6.33 \%$ to $14.23 \%$ in SNB19 cells (Figure 1B). These results indicated that rottlerin stimulated cell apoptosis in glioma cells.

\section{Rottlerin induced cell cycle arrest in glioma cells}

To dissect whether rottlerin treatment could arrest cell cycle in glioma cells, cell cycle analysis with PI staining and flow cytometry was used to test the cell cycle in U251 and SNB19 cells after $2 \mu \mathrm{M}$ and $4 \mu \mathrm{M}$ rottlerin treatments for $48 \mathrm{~h}$. We found that it has a typical $\mathrm{G}_{0} / \mathrm{G}_{1}$ cycle arrest in the rottlerin-treated glioma cells (Figure 1C). Specifically, $2 \mu \mathrm{M}$ and $4 \mu \mathrm{M}$ rottlerin led to G1 cell population from $59 \%$ to $77 \%$ to $82 \%$, respectively, in U251 cells (Figure 1C). Similarly, $2 \mu \mathrm{M}$ and $4 \mu \mathrm{M}$ rottlerin treatments caused $\mathrm{G} 1$ cell population from $65.7 \%$ to $73.4 \%$ to $81 \%$, respectively, in SNB19 cells (Figure 1C). These findings suggest that rottlerin induced G1 cell cycle arrest in glioma cells.

\section{Rottlerin inhibited cell migration and invasion}

To explore whether rottlerin plays a key role in regulation of motility of glioma cells, invasion assay was performed in glioma cells treated with rottlerin for 20 hours. We found that rottlerin significantly retarded the penetration of glioma cells through the Matrigel-coated membrane in a dose-dependent manner, suggesting that rottlerin inhibited cell invasion in glioma cells (Figure 2A). To further investigate the inhibition of migration in glioma cells by rottlerin, scratch assay was used to detect the migratory activity in glioma cells after rottlerin treatment. The results from our wound healing 
assay showed that rottlerin remarkably suppressed cell migration in a dose-dependent manner in both glioma cell lines (Figure 2B). Altogether, rottlerin inhibited cell motility in glioma cells.

\section{Rottlerin decreased Cdc20 expression}

Emerging evidence has demonstrated that $\mathrm{Cdc} 20$ plays an oncogenic role in the development and progression of human cancers [18]. Further studies revealed that targeting $\mathrm{Cdc} 20$ could be a novel therapeutic approach in human cancers [29]. Therefore, we explored whether rottlerin could down-regulate the expression of Cdc20 in glioma cells. RT-PCR and Western blotting analysis were used to detect the expression of Cdc20 in glioma cells after rottlerin treatment for 72 hours. Our RT-PCR results showed that Cdc20 mRNA level was down-regulated by rottlerin in U251 and SNB19 cells (Figure 3A). Moreover, our Western blotting analysis revealed that rottlerin significantly decreased Cdc20 protein levels in glioma cells (Figure 3B and 3C). Importantly, we also found that p21, one of Cdc20 targets, was increased in both glioma
A

U251 cells

$48 \mathrm{~h}$

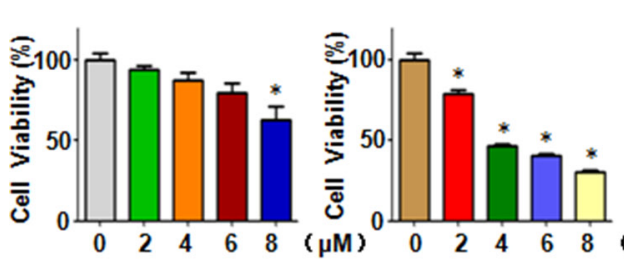

B

U251 cells

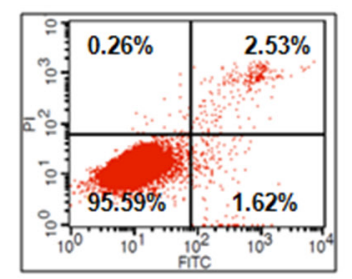

Control

C

SNB19 cells

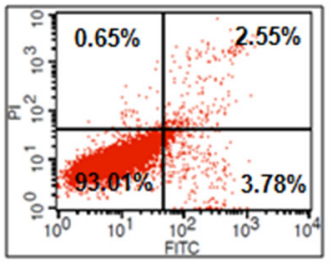

Control

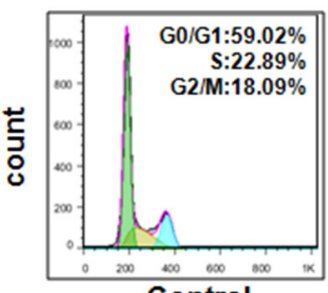

Control

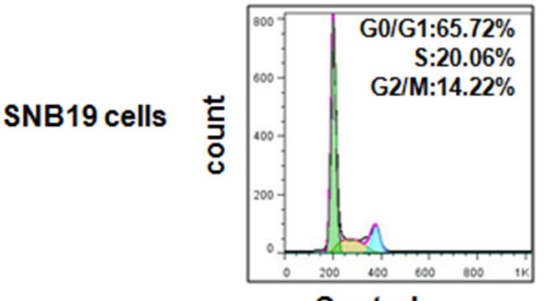

Control

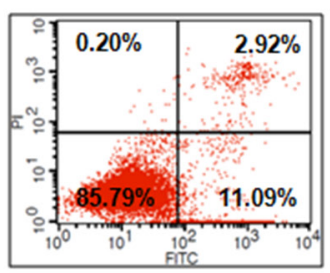

$2 \mu \mathrm{M}$

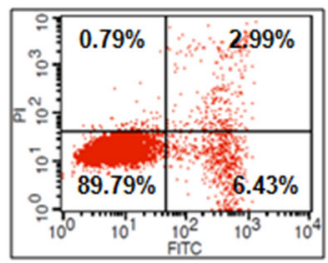

$2 \mu \mathrm{M}$
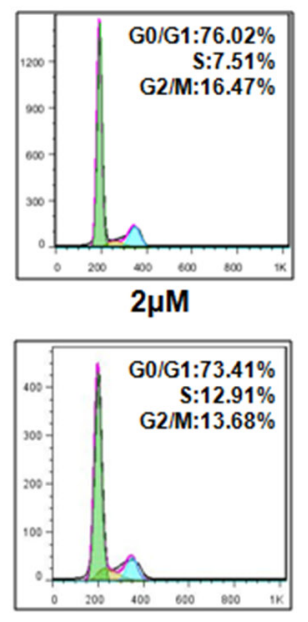

$2 \mu \mathrm{M}$
$72 \mathrm{~h}$

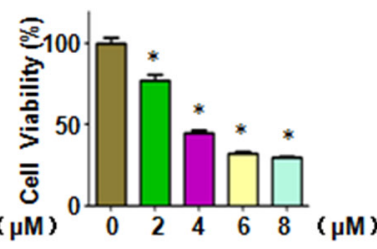

$4 \mu \mathrm{M}$
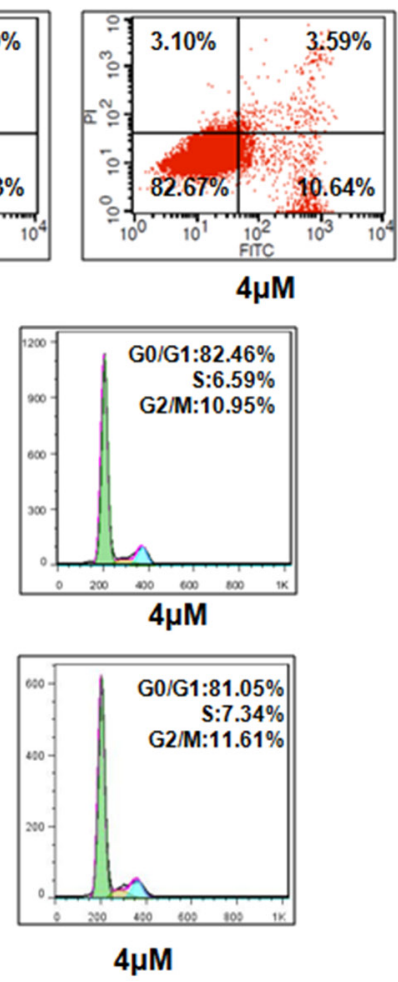

Figure 1: Effect of Rottlerin on cell growth, apoptosis, and cell arrest. (A) MTT assay was used to detect the cell growth in glioma cells treated with rottlerin. ${ }^{*} P<0.05$, compared to the control. (B) Cell apoptosis was conducted by FACS in glioma cells treated with rottlerin. (C) Cell cycle analysis was determined by Flow cytometry in rottlerin-treated glioma cells. 
cells after rottlerin treatment (Figure 3B and 3C). Our findings demonstrated that rottlerin could be a potential inhibitor of Cdc20 in glioma cells.

\section{Over-expression of $\mathrm{Cdc20}$ reversed the anti- proliferation of rottlerin}

To validate the function of $\mathrm{Cdc} 20$ in rottlerinmediated cell growth inhibition, Cdc20 cDNA or empty vector as control was transfected into glioma cells followed by rottlerin treatment for 48 hours. We found that overexpression of $\mathrm{Cdc} 20$ promoted cell growth in both glioma cells (Figure 4A). More importantly, we observed that $\mathrm{Cdc} 20$ overexpression abrogated cell growth inhibition induced by rottlerin in glioma cells (Figure 4A). These findings indicated that rottlerin exerts its anti-proliferation of glioma cells via inhibition of Cdc20 in glioma cells.

\section{Over-expression of Cdc20 abrogated anti- motility of rottlerin in glioma cells}

To measure the effect of Cdc20 overexpression on cell invasion, Matrigel invasion assay was conducted in glioma cells after rottlerin treatment and Cdc20 cDNA transfection. We found that overexpression of Cdc20 significantly enhanced cell invasion in both U251 cells and SNB19 cells (Figure 4B). Moreover, Cdc20 overexpression abrogated inhibition of cell invasion induced by rottlerin in glioma cells (Figure 4B). To further explore whether Cdc20 could control cell motility in glioma cell lines, scratch assay was performed in glioma
A U251 cells

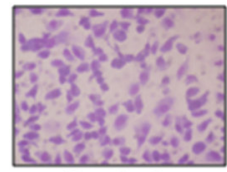

Control

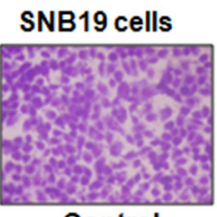

Control

B
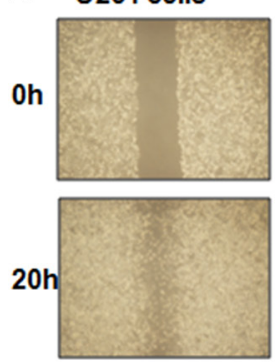

Control

SNB19 cells

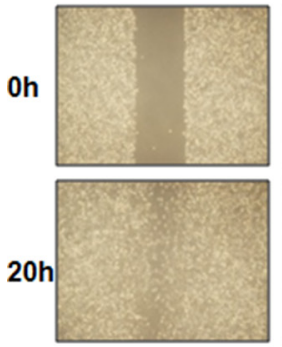

Control

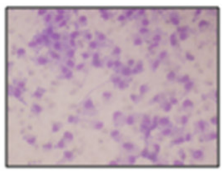

$2 \mu \mathrm{M}$

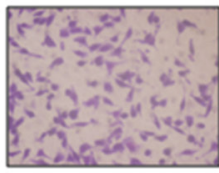

$2 \mu \mathrm{M}$
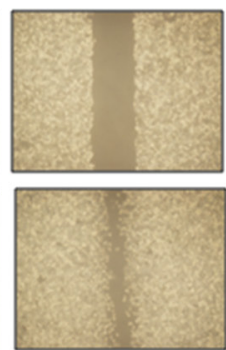

$2 \mu \mathrm{M}$
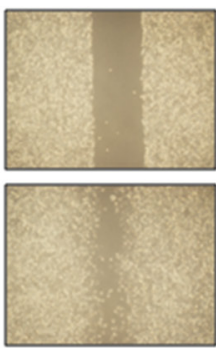

$2 \mu \mathrm{M}$
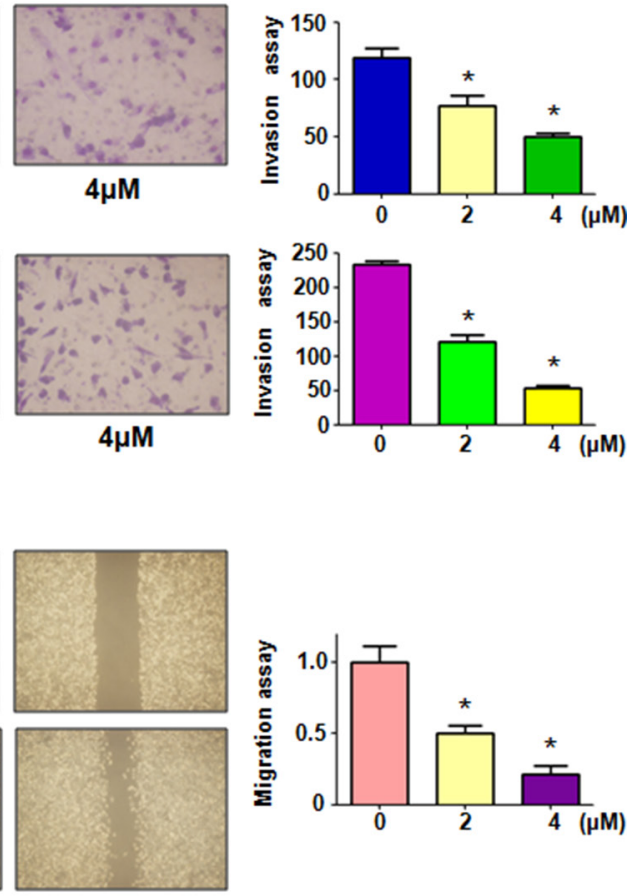

$4 \mu \mathrm{M}$
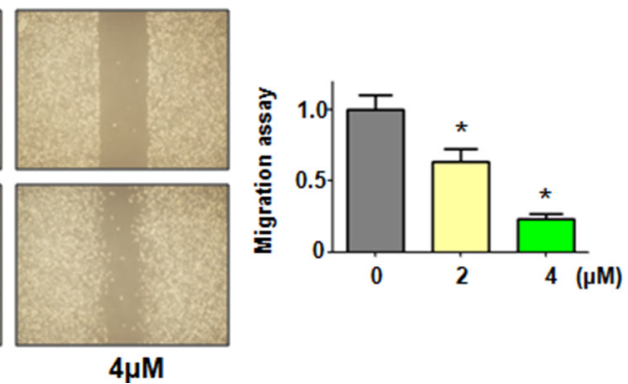

Figure 2: Rottlerin inhibited cell migration and invasion in glioma cells. (A) Left panel: The inhibitory effect of rottlerin on glioma cell invasion was determined by Transwell chambers assay in U251 cells (Top panel) and SNB19 cells (Bottom panel). Right panel: Quantitative results are illustrated for left panel. (B) Left panel: Cell migration was detected using wound healing assay in U251 cells (Top panel) and SNB19 cells (Bottom panel) after rottlerin treatment. Quantitative results are illustrated for left panel. $* P<0.05, * * P<0.01$ vs control. 
cells treated with rottlerin in combination with $\mathrm{Cdc} 20$ cDNA transfection. Our scratch assay showed that overexpression of $\mathrm{Cdc} 20$ promoted the glioma cell migration (Figure 5A). Overexpression of Cdc20 reversed the cell migration inhibition in rottlerin-treated glioma cells (Figure 5A). Importantly, we found that Cdc20 cDNA transfection upregulated $\mathrm{Cdc} 20$ expression in glioma cells (Figure 5B). Additionally, Cdc20 overexpression abrogated the inhibition of $\mathrm{Cdc} 20$ in glioma cells treated with rottlerin (Figure 5B). Consistently, p21 expression is downregulated in Cdc20-transfected glioma cells (Figure 5B). Similarly, Cdc20 cDNA transfection reversed the upregulation of $\mathrm{p} 21$ by rottlerin in both glioma cells (Figure 5B). Our data suggested that rottlerin inhibited cell motility partly through inhibition of Cdc20 in glioma cells.

\section{Down-regulation of Cdc20 by its shRNA promoted rottlerin-induced antitumor activity}

To further determine the role of Cdc20 in rottlerinmediated tumor suppressive activity, we depleted Cdc20 by its shRNA in glioma cells. We found that downregulation of Cdc20 inhibited cell growth in both glioma cells (Figure 6A). Moreover, depletion of Cdc20 enhanced cell growth inhibition induced by rottlerin in glioma cells (Figure 6A). We also observed that down-regulation of Cdc20 triggered cell apoptosis in glioma cells (Figure 6B). Cdc20 shRNA treatment led to more apoptotic cells induced by rottlerin compared with rottlerin alone or $\mathrm{Cdc} 20$ shRNA transfection alone in glioma cells (Figure 6B). Our invasion assay showed that depletion of Cdc20 suppressed
A U251 cells

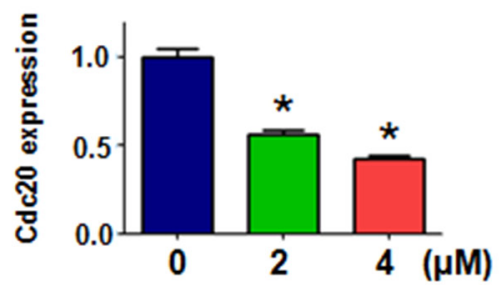

B

$$
\text { U251 cells }
$$

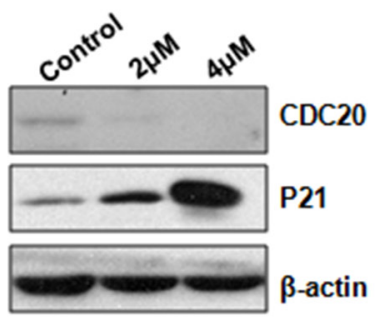

C
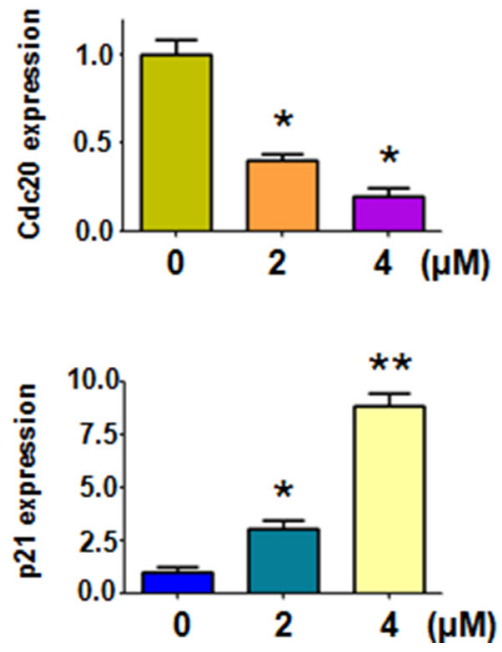

\section{SNB19 cells}

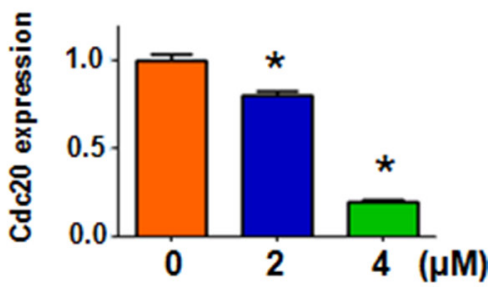

SNB19 cells
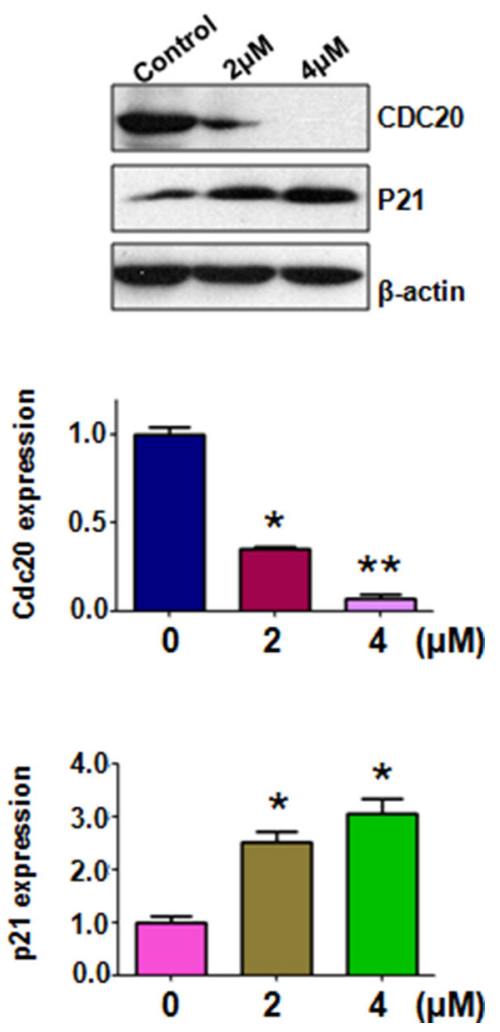

Figure 3: Rottlerin downregulated Cdc20 expression at RNA and protein levels. (A) The mRNA level of Cdc20 was measured using RT-PCR in glioma cells treated with rottlerin. ${ }^{*} P<0.05, * * P<0.01$ vs control. (B) The expression of Cdc20 and p21 was determined by Western blotting analysis in U251 and SNB19 cells after rottlerin treatment. (C) Quantitative results are illustrated for left panel. $* P<0.05, * * P<0.01$, compared to the control. 
glioma cell invasion (Figure 6C). Notably, Cdc20 shRNA enhanced cell invasion inhibition in rottlerin-treated glioma cells (Figure 6C). In line with this, depletion of Cdc20 retarded the cell migration in both U251 and SNB19 cells (Figure 7A). Furthermore, Cdc20 shRNA promoted inhibition of migration induced by rottlerin in glioma cells (Figure 7A). Our Western blotting analysis demonstrated that $\mathrm{Cdc} 20$ shRNA enhanced rottlerin-mediated inhibition of Cdc20 in glioma cells (Figure 7B). Consistently, Cdc20 shRNA promoted rottlerin-induced p21 level in both glioma cells (Figure 7B).

\section{DISCUSSION}

Rottlerin increased HO-1 (heme oxygenase-1) expression via ROS (reactive oxygen species) pathway in human colon cancer cells [30]. Rottlerin was also exhibited its anti-angiogenesis function via inhibition of human microvascular endothelial cell proliferation and tube formation on Matrigel [31]. Moreover, rottlerin was reported to enhance IL-1 $\beta$ (interleukin-1 $\beta$ )-induced COX-2 (cyclooxygenase-2) expression via activation of p38 MAPK (mitogen-activated protein kinases) in breast cancer cells [32]. Lim et al found that rottlerin induced apoptosis via upregulation of NAG-1 through ERK (extracellular signal regulated kinase) and p38 MAPK pathway in colon cancer cells [32]. Furthermore, rottlerin inhibited PI3K (phosphoinositide 3-kinase)/Akt/mTOR (mammalian target of rapamycin) pathway, leading to autophagy and apoptosis in pancreatic cancer stem cells [33] and prostate cancer cells [34]. Lu et al. reported that rottlerin induced LRP6 (LDL-receptor related protein 6) degradation and inhibited both $\mathrm{Wnt} / \beta$-catherin and mTORC1 signaling in prostate and breast cancer cells [35]. Recently, it has been reported that rottlerin inhibited pancreatic tumor growth in nude mice [36]. Notably, rottlerin suppressed cell growth via the dual inhibition of ERK and NF- $\mathrm{KB}$ and downregulation of Cyclin D1 in melanoma cells [37]. In the present study, we identified that rottlerin inhibited Cdc20 expression, resulting in inhibition of tumorigenesis in glioma.

Recently, Cdc20 was reported to be a key factor in maintaining tumorigenic glioma tumor initiating cells through degradation of $\mathrm{p} 21$ and regulation of $\mathrm{Cdc} 25 \mathrm{C}$ (cell division cycle 25C), c-Myc, and Survivin [38]. In support of this concept, another study demonstrated that Cdc20 drives invasiveness and self-renewal in patient tumor-derived GSCs (glioblastoma stem-like cells) through pluripotenty related transcription factor SOX2 [39]. Moreover, using an orthotopic xenograft model overexpression of Cdc20 enhanced the GSCs to generate brain tumors, whereas depletion of Cdc20 retarded the
A

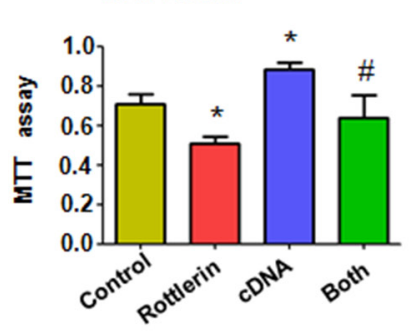

B U251 cells

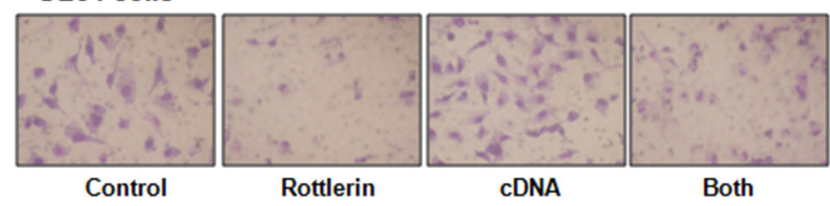

SNB19 cells

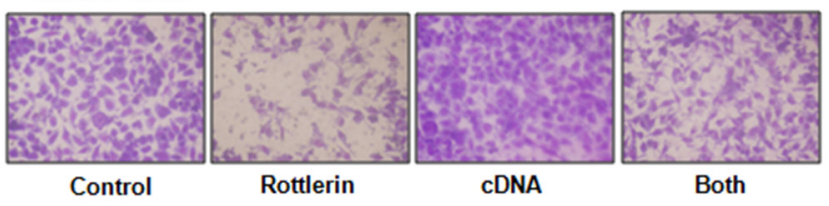

SNB19 cells
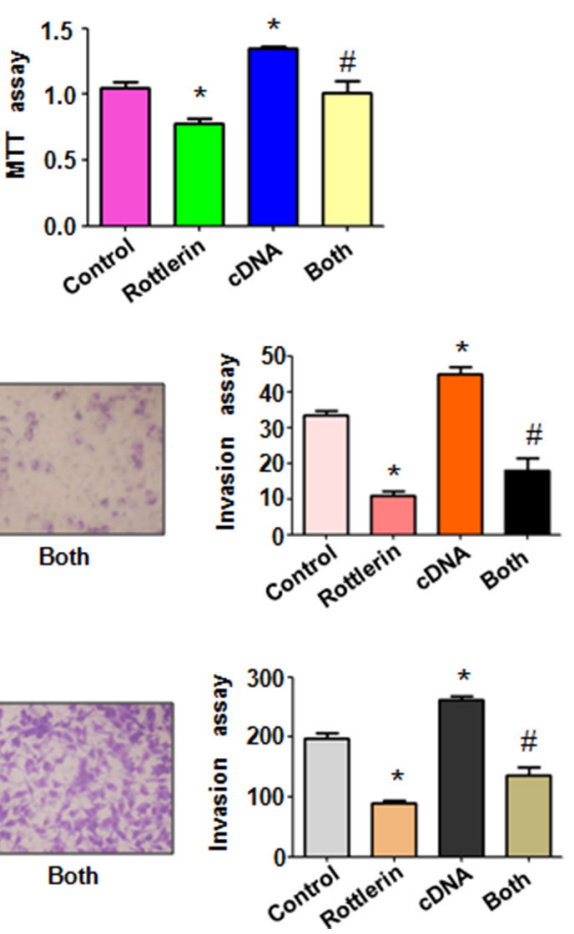

Figure 4: The effect of Cdc20 overexpression on cell growth and invasion. (A) MTT assay was used to detect the effect of Cdc20 overexpression in combination with rottlerin treatment on glioma cell proliferation. Control: pcDNA 3.1 treatment; Rottlerin: $2 \mu \mathrm{M}$ rottlerin; cDNA: Cdc20 cDNA; Both: rottlerin + Cdc20 cDNA. ${ }^{*} P<0.05,{ }^{* *} P<0.01$, compared with control; ${ }^{\#} P<0.05$ compared with rottlerin treatment or Cdc20 cDNA transfection. (B) Left panel, Invasion assay was performed in glioma cells after Cdc20 cDNA transfection and rottlerin treatment. Right panel, Quantitative results are illustrated for left panel. 
GSCs to develop brain tumors [39]. Strikingly, it has been reported that $\mathrm{Cdc} 20$ induced the proliferation of primary glial progenitor cells [40]. Some studies showed that rottlerin inhibited cancer cell growth and induced apoptosis in a variety of human cancers [13, 14, 41, 42]. Moreover, rottlerin could suppress growth of human pancreatic tumors in nude mice $[43,44]$. We also found that overexpression of $\mathrm{Cdc} 20$ enhanced glioma cell proliferation, whereas depletion of Cdc20 suppressed cell growth in glioma cells. Our results further showed that $\mathrm{Cdc} 20$ overexpression enhanced cell migration and invasion in glioma cells, while downregulation of Cdc20 retarded cell motility. Altogether, Cdc20 plays a pivotal role in regulation of cell growth, migration and invasion in glioma cells.

Since Cdc20 is identified as an oncoprotein in tumorigenesis, inactivation of Cdc20 could be useful for the treatment of human cancers. To this end, several Cdc20
A

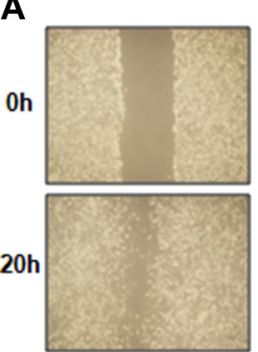

Control
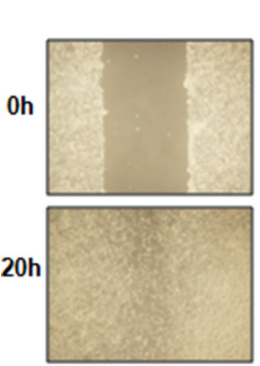

Control

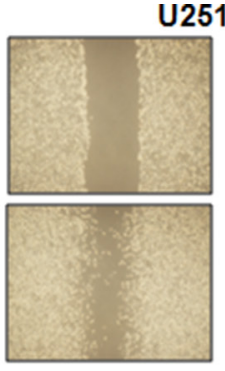

Rottlerin

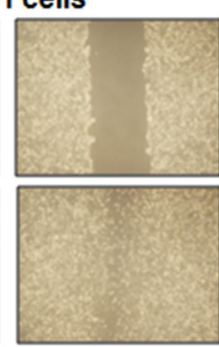

cDNA
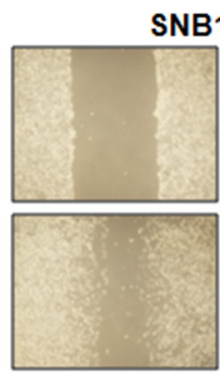

Rottlerin
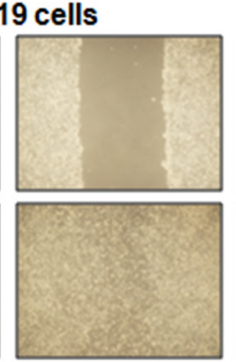

cDNA

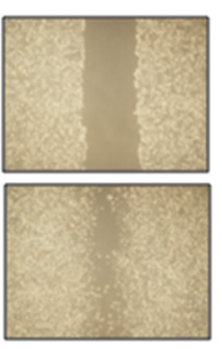

Both
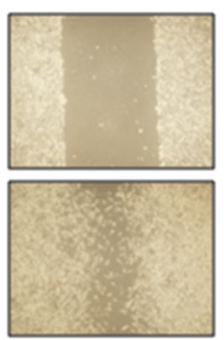

Both
U251

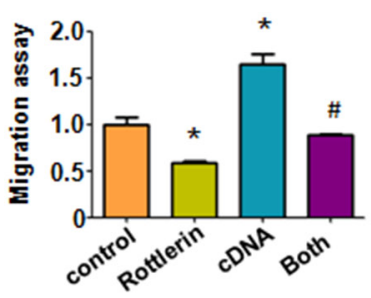

\section{SNB19}

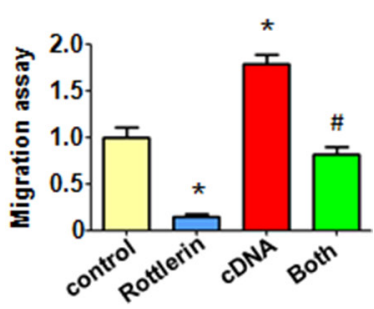

B
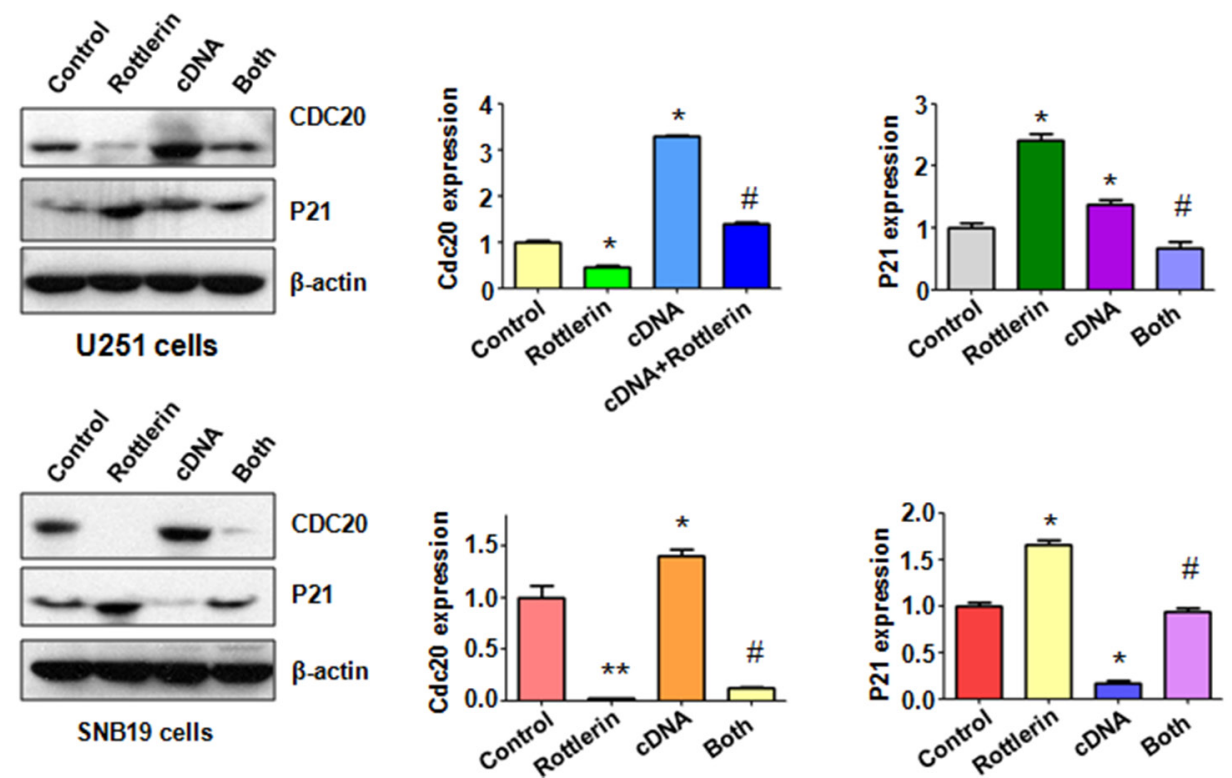

Figure 5: The effect of Cdc20 overexpression on cell migration in glioma cells. (A) Left panel: The wound healing assay was conducted to detect the cell migration in glioma cells after Cdc20 cDNA transfection and rottleirn treatment. Right panel: Quantitative results are illustrated for left panel. Control: pcDNA 3.1 treatment; Rottlerin: $2 \mu \mathrm{M}$ rottlerin; cDNA: Cdc20 cDNA; Both: rottlerin + Cdc20 cDNA. $* P<0.05,{ }^{* *} P<0.01$, compared with control; ${ }^{*} P<0.05$ compared with rottlerin treatment or Cdc20 cDNA transfection. (B) Left panel: The expression of Cdc20 and its target p21 was detected by western blotting in glioma cells with Cdc20 cDNA transfection and rottlerin treatment. Right panel: Quantitative results are illustrated for left panel. 
inhibitors have been discovered. For example, TAME (tosyl-L-arginine methyl ester) reduced Cdc20 association with the APC and subsequent inhibited APC E3 ligase activity [45]. Moreover, pro-TAME with cell permeable activity disrupted the APC-Cdc20/Cdh1 interaction to reduce $\mathrm{APC}$ activation, leading to cell death in multiple cancer cell lines [45]. Notably, apcin (APC inhibitor) binds Cdc20 and prevents substrate recognition and competitively suppresses the ubiquitination of Cdc20 substrates [46]. Strikingly, apcin and pro-TAME synergized to enhance the mitotic fraction in human cancer cell lines [46]. In addition, compound 331 was found to be a potential drug selectively targeting glioma cells through down-regulation of Cdc20 [47]. NAHA, a N-alkylated amino acid-derived sulfonamide hydroxamate, inhibited the expression of Cde20 in breast cancer cells [48]. Several natural agents were identified to inhibit the $\mathrm{Cdc} 20$ expression in human cancer cells. withaferin A exerted its anti-cancer activity through enhanced degradation of Cdc20 and Mad2 [49]. Ganodermanontriol (GDNT), a ganoderma
A

U251 cells

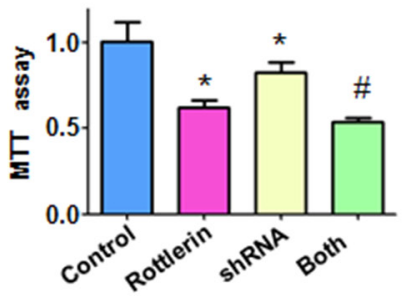

B

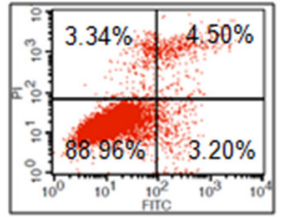

Control SNB19 cells

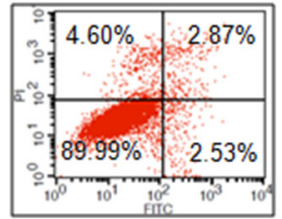

Control

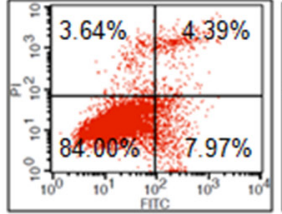

Rottlerin

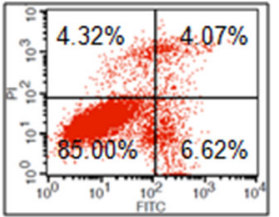

Rottlerin

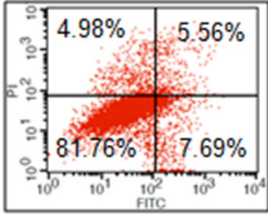

shRNA

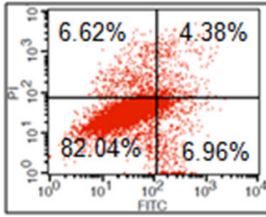

shRNA

C U251 cells

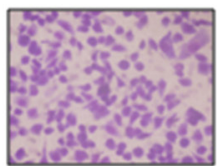

Control

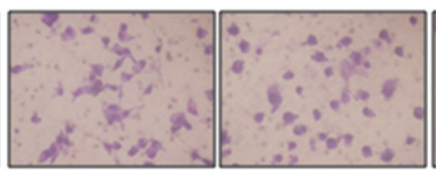

Rottlerin

shRNA

\section{SNB19 cells}

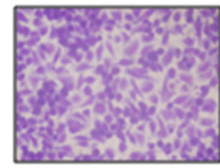

Control

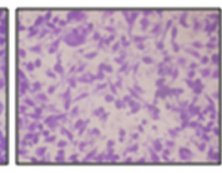

Rottlerin

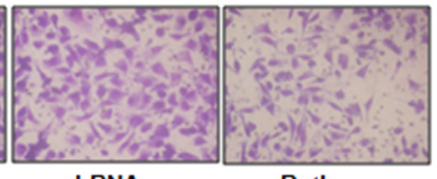

shRNA

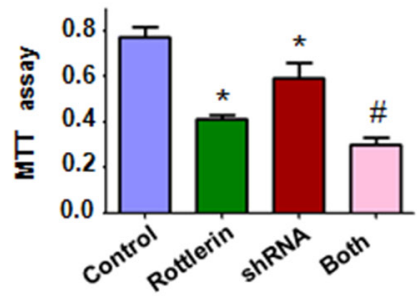

SNB19 cells

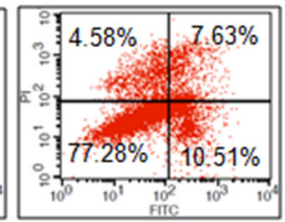

Both

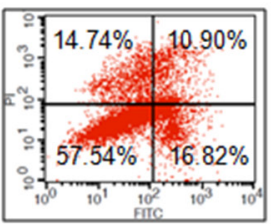

Both
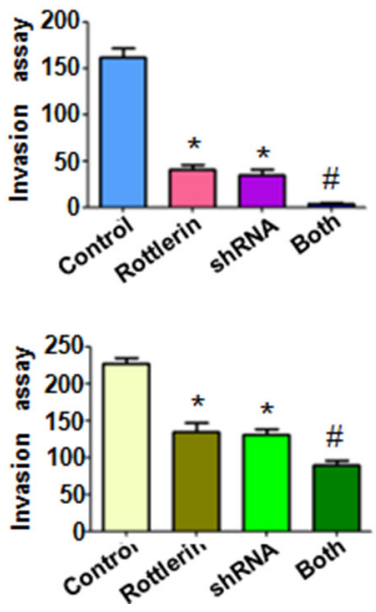

Figure 6: The effect of Cdc20 downregulation on cell growth, apoptosis and invasion. (A) MTT assay was used to detect the effect of Cdc20 shRNA in combination with rottlerin treatment on glioma cell proliferation. Control: siRNA control; Rottlerin: $2 \mu \mathrm{M}$ rottlerin; shRNA: Cdc20 shRNA; Both: rottlerin + Cdc20 shRNA. ${ }^{*} P<0.05$, compared with control; ${ }^{\#} P<0.05$ compared with rottlerin treatment or Cdc20 shRNA. (B) Apoptosis was detected by Flow cytometry in glioma cells with Cdc20 shRNA and rottlerin treatment. (C) Invasion assay was performed in glioma cells after Cdc20 shRNA and rottlerin treatment. 
alcohol from medicinal mushroom, inactivated Cdc20 in breast cancer cells [50]. Genistein, the predominant isoflavone in soybean enriched foods, was reported to exert its anti-carcinogenic properties through inhibition of multiple genes including Cdc20 in primary glioblastoma, rhabdomyosarcoma, hepatocellular carcinoma and human embryonic carcinoma cells [51]. Moreover, genistein controlled Cdc20 expression, leading to regulation of cell cycle in breast cancer cells [52]. Here, we identified that rottlerin could be a new Cdc20 inhibitor in glioma cells. Due to non-toxic nature, inactivation of $\mathrm{Cdc} 20$ by rottlerin could be a safer strategy for the treatment of glioma.
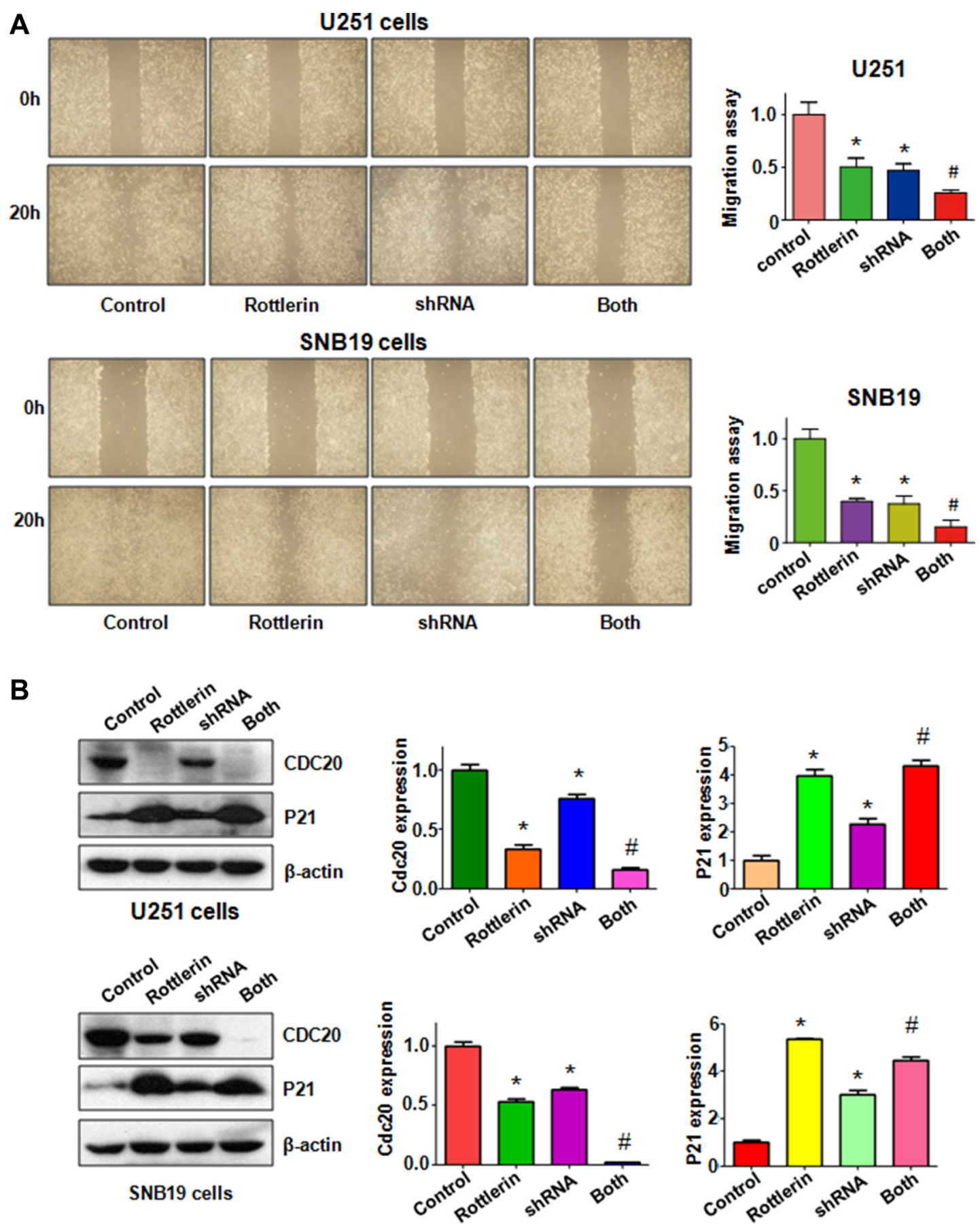

Figure 7: The effect of Cdc20 downregulation on cell migration in glioma cells. (A) Left panel: The wound healing assay was conducted to detect the cell migration in glioma cells after Cdc20 shRNA and rottlerin treatment. Control: siRNA control; Rottlerin: $2 \mu \mathrm{M}$ rottlerin; shRNA: Cdc20 shRNA; Both: rottlerin + Cdc20 shRNA. Right panel: Quantitative results are illustrated for left panel. $* P<0.05$, vs control; ${ }^{\#} P<0.05$ vs Cdc20 shRNA treatment or rottlerin treatment. (B) Left panel: The expression of Cdc20 and its target p21 was detected by western blotting in glioma cells with Cdc20 shRNA and rottlerin treatment. Right panel: Quantitative results are illustrated for left panel. $* P<0.05,{ }^{* *} P<0.01$, compared with control; ${ }^{\#} P<0.05$ compared with rottlerin treatment or Cdc20 shRNA treatment. 


\section{MATERIALS AND METHODS}

\section{Cell culture and experimental regents}

Human glioma U251 cells and SNB19 cells were cultured in DMEM medium with $10 \%$ fetal bovine serum and $1 \%$ penicillin and streptomycin in a $5 \% \mathrm{CO} 2$ at $37^{\circ} \mathrm{C}$. Primary antibodies for $\mathrm{Cdc} 20$ (\#14866, 1:1000) and p21 (\#2947, 1:1000) were purchased from Abcam and Cell Signaling Technology, respectively. All secondary antibodies were purchased from Thermo Scientific. Lipofectamine 2000 was purchased from Invitrogen. Monoclonal anti- $\beta$-actin (A2228, 1:5000), rottlerin (CAS number 82-08-6, 85\%), and MTT (3-4,5-dimethyl-2thiazolyl-2, 5-diphenyl-2-H-tetrazolium bromide) were obtained from Sigma-Aldrich (St.Louis, MO).

\section{MTT assay}

Cells were seeded in 96-well plate at a density of $5 \times 10^{3}$ cells/well in a volume of $100 \mu \mathrm{l}$ per well for $24 \mathrm{~h}$ and treated with different concentrations of rottlerin. After $48 \mathrm{~h}$ and $72 \mathrm{~h}, 10 \mu \mathrm{l}$ MTT solution $(0.5 \mathrm{mg} / \mathrm{ml})$ was added to each well, and the plates were incubated for $4 \mathrm{~h}$ at $37^{\circ} \mathrm{C}$ with $5 \% \mathrm{CO}_{2}$. Then, the liquid supernatant was removed and $100 \mu \mathrm{l}$ DMSO was added in each well. The absorption was measured at $490 \mathrm{~nm}$ on the Multimode Reader of SpectraMax M5 (Moleucular Devices, US).

\section{Cell apoptosis assay}

Cells were seeded at a density of $2 \times 10^{5}$ cells/well in 6-well plate with different concentration of rottlerin. After $48 \mathrm{~h}$, cells were harvested and washed with PBS, cells were suspended with $500 \mu \mathrm{l}$ binding buffer with $5 \mu \mathrm{l}$ FITC-conjugated anti-Annexin V antibody and $5 \mu \mathrm{PI}$ (Propidium iodide), and then analyzed with a FACScalibur flow cytometer (BD, USA).

\section{Cell cycle analysis}

Cells were cultured in six-well plate at a density of $2 \times 10^{5}$ cells/well overnight and treated with various concentration of rottlerin. After $48 \mathrm{~h}$, cells were harvested and washed with PBS, and suspended with $70 \%$ cold alcohol for overnight in $4^{\circ} \mathrm{C}$ icebox. Then, cells were washed and suspended with $500 \mu \mathrm{PBS}$. Then incubated with $100 \mu \mathrm{g} / \mathrm{ml}$ RNase and $50 \mathrm{mg} / \mathrm{ml}$ PI (Propidium iodide) at $37^{\circ} \mathrm{C}$ and then analyzed the cell cycle with a FACScalibur flow cytometer (BD, USA).

\section{Cell scratch assay}

Cells were seeded in 6-well plate for overnight and reached almost $100 \%$ confluence. Then scratch the cells with a sterile $20 \mu \mathrm{l}$ pipette tip. Wash the detached cells with PBS and incubate with different concentrations of rottlerin. The scratch was photographed with Box-Type Fluorescence Imaging Device (FSX100, OLYMPUS) at $0 \mathrm{~h}$ and $20 \mathrm{~h}$.

\section{Cell invasion assay}

Cell invasion assay was used to determine the invasive activity of glioma cells treated with rottlerin or Cdc20 cDNA or shRNA. Cells tranfected with Cdc20 cDNA or shRNA incubated with rottlerin or not with $200 \mu \mathrm{l}$ serum-free medium in the up camber with Matrigel. Then added $500 \mu \mathrm{l}$ DMEM medium with $10 \%$ FBS. The cells were incubated for $20 \mathrm{~h}$ at $37^{\circ} \mathrm{C}$ with $5 \%$ $\mathrm{CO}_{2}$ and then strained with Giemsa and photographed with Box-Type Fluorescence Imaging Device (FSX100, OLYMPUS).

\section{Quantitative real-time reverse transcription- PCR analysis}

The total RNA was extracted with Trizol (Invitrogen, Carlsbad, CA) and reversed-transcribed into cDNA by RevertAid First Strand cDNA Synthesis Kit. PCR were performed using Power SYBR Green PCR Master Mix and the results were calculated by $2-\Delta \Delta \mathrm{Ct}$ method. The primers used in the PCR reaction are: $\mathrm{Cdc} 20$, forward primer (5'- GAC CAC TCC TAG CAA ACC TGG -3') and reverse primer (5'-GGG CGT CTG GCT GTT TTC A-3'); GAPDH, forward primer (5'- ACC CAG AAG ACT GTG GAT GG - $3^{\prime}$ ) and reverse primer (5'- CAG TGA GCT TCC CGT TCA G- 3').

\section{Western blotting analysis}

The harvested cells were washed by PBS and lysed with protein lysis buffer. The concentrations of the proteins were tested by BCA Protein Assay kit (Thermo Scientific, MA). Same amount of protein samples were separated by electrophoresis in Sodium Dodecyl Sulfonate (SDS)-polyacrylamide gel and then transferred onto a Polyvinylidene Fluoride (PVDF) membrane, and then incubated with primary antibody at $4^{\circ} \mathrm{C}$ overnight. After washed with TBST for three times and incubated with second antibody at room temperature for one hour. Then the expression of protein was detected by electrochemiluminescence (ECL) assay [53].

\section{Transfection}

Cells were seeded into 6-well plates and transfected with Cdc20 cDNA or Cdc20 shRNA or empty vector using lipofectamine 2000 following the instruction's protocol. After the transfection, the cells were subjected to further analysis as described under the results sections. 


\section{Statistical analysis}

All statistical analyses were conducted using GraphPad Prism 4.0 (Graph Pad Software, La Jolla, CA). Student's t-test was performed to evaluate statistical significance. $P<0.05$ was considered as statistically significant.

\section{ACKNOWLEDGMENTS}

This work was supported by grant from National Natural Science Foundation of China (NSFC number 81572936) and the priority academic program development of Jiangsu higher education institutions.

\section{CONFLICTS OF INTEREST}

There is no conflicts of interest.

\section{REFERENCES}

1. Siegel RL, Miller KD, Jemal A. Cancer statistics, 2016. CA Cancer J Clin. 2016; 66:7-30.

2. Aibaidula A, Lu JF, Wu JS, Zou HJ, Chen H, Wang YQ, Qin ZY, Yao Y, Gong Y, Che XM, Zhong P, Li SQ, Bao WM, et al. Establishment and maintenance of a standardized glioma tissue bank: Huashan experience. Cell Tissue Bank. 2015; 16:271-281.

3. Alifieris C, Trafalis DT. Glioblastoma multiforme: Pathogenesis and treatment. Pharmacol Ther. 2015.

4. Ostrom QT, Gittleman H, Stetson L, Virk SM, BarnholtzSloan JS. Epidemiology of gliomas. Cancer Treat Res. 2015; 163:1-14.

5. Walbert T, Chasteen K. Palliative and supportive care for glioma patients. Cancer Treat Res. 2015; 163:171-184.

6. Dai XJ, Jiang WJ, Wang WM, Zhao SJ. Drug or vaccine?: selecting the appropriate treatment for malignant glioma patients. Drugs. 2010; 70:1477-1486.

7. Hasima N, Ozpolat B. Regulation of autophagy by polyphenolic compounds as a potential therapeutic strategy for cancer. Cell Death Dis. 2014; 5:e1509.

8. Maioli E, Torricelli C, Valacchi G. Rottlerin and cancer: novel evidence and mechanisms. ScientificWorld Journal. 2012; 2012:350826.

9. Maioli E, Greci L, Soucek K, Hyzdalova M, Pecorelli A, Fortino V, Valacchi G. Rottlerin inhibits ROS formation and prevents NFkappaB activation in MCF-7 and HT-29 cells. J Biomed Biotechnol. 2009; 2009:742936.

10. Lin CJ, Lin CY, Chen Y, Huang SH, Wang SM. Rottlerin inhibits migration of follicular thyroid carcinoma cells by PKCdelta-independent destabilization of the focal adhesion complex. J Cell Biochem. 2010; 110:428-437.

11. Ohno I, Eibl G, Odinokova I, Edderkaoui M, Damoiseaux RD, Yazbec M, Abrol R, Goddard WA, 3rd, Yokosuka O, Pandol SJ, Gukovskaya AS. Rottlerin stimulates apoptosis in pancreatic cancer cells through interactions with proteins of the Bcl-2 family. Am J Physiol Gastrointest Liver Physiol. 2010; 298:G63-73.

12. Jain SK, Pathania AS, Meena S, Sharma R, Sharma A, Singh B, Gupta BD, Bhushan S, Bharate SB, Vishwakarma RA. Semisynthesis of mallotus B from rottlerin: evaluation of cytotoxicity and apoptosis-inducing activity. J Nat Prod. 2013; 76:1724-1730.

13. Song KS, Kim JS, Yun EJ, Kim YR, Seo KS, Park JH, Jung YJ, Park JI, Kweon GR, Yoon WH, Lim K, Hwang BD. Rottlerin induces autophagy and apoptotic cell death through a PKC-delta-independent pathway in HT1080 human fibrosarcoma cells: the protective role of autophagy in apoptosis. Autophagy. 2008; 4:650-658.

14. Lim JH, Park JW, Choi KS, Park YB, Kwon TK. Rottlerin induces apoptosis via death receptor 5 (DR5) upregulation through CHOP-dependent and PKC deltaindependent mechanism in human malignant tumor cells. Carcinogenesis. 2009; 30:729-736.

15. Torricelli C, Fortino V, Capurro E, Valacchi G, Pacini A, Muscettola M, Soucek K, Maioli E. Rottlerin inhibits the nuclear factor kappaB/cyclin-D1 cascade in MCF-7 breast cancer cells. Life Sci. 2008; 82:638-643.

16. Ohno I, Eibl G, Odinokova I, Edderkaoui M, Damoiseaux RD, Yazbec M, Abrol R, Goddard WA, 3rd, Yokosuka O, Pandol SJ, Gukovskaya AS. Rottlerin stimulates apoptosis in pancreatic cancer cells through interactions with proteins of the Bcl-2 family. Am J Physiol Gastrointest Liver Physiol. 2010; 298:G63-73.

17. Weissman AM, Shabek N, Ciechanover A. The predator becomes the prey: regulating the ubiquitin system by ubiquitylation and degradation. Nat Rev Mol Cell Biol. 2011; 12:605-620.

18. Wang L, Zhang J, Wan L, Zhou X, Wang Z, Wei W. Targeting Cdc20 as a novel cancer therapeutic strategy. Pharmacol Ther. 2015; 151:141-151.

19. Zhang S, Chang L, Alfieri C, Zhang Z, Yang J, Maslen S, Skehel M, Barford D. Molecular mechanism of APC/C activation by mitotic phosphorylation. Nature. 2016; 533:260-264.

20. Fujimitsu K, Grimaldi M, Yamano H. Cyclin-dependent kinase 1-dependent activation of APC/C ubiquitin ligase. Science. 2016; 352:1121-1124.

21. Kim Y, Choi JW, Lee JH, Kim YS. MAD2 and CDC20 are upregulated in high-grade squamous intraepithelial lesions and squamous cell carcinomas of the uterine cervix. Int $\mathrm{J}$ Gynecol Pathol. 2014; 33:517-523.

22. Moura IM, Delgado ML, Silva PM, Lopes CA, do Amaral JB, Monteiro LS, Bousbaa H. High CDC20 expression is associated with poor prognosis in oral squamous cell carcinoma. J Oral Pathol Med. 2014; 43:225-231.

23. Wu WJ, Hu KS, Wang DS, Zeng ZL, Zhang DS, Chen DL, Bai L, Xu RH. CDC20 overexpression predicts a poor 
prognosis for patients with colorectal cancer. J Transl Med. 2013; 11:142.

24. Marucci G, Morandi L, Magrini E, Farnedi A, Franceschi E, Miglio R, Calo D, Pession A, Foschini MP, Eusebi V. Gene expression profiling in glioblastoma and immunohistochemical evaluation of IGFBP-2 and CDC20. Virchows Archiv. 2008; 453:599-609.

25. Bie L, Zhao G, Cheng P, Rondeau G, Porwollik S, Ju Y, Xia XQ, McClelland M. The accuracy of survival time prediction for patients with glioma is improved by measuring mitotic spindle checkpoint gene expression. PloS one. $2011 ; 6: \mathrm{e} 25631$.

26. Amador V, Ge S, Santamaria PG, Guardavaccaro D, Pagano M. APC/C(Cdc20) controls the ubiquitin-mediated degradation of p21 in prometaphase. Mol Cell. 2007; 27:462-473

27. Hadjihannas MV, Bernkopf DB, Bruckner M, Behrens J. Cell cycle control of Wnt/beta-catenin signalling by conductin/axin2 through CDC20. EMBO Rep. 2012; 13:347-354.

28. Kidokoro T, Tanikawa C, Furukawa Y, Katagiri T, Nakamura Y, Matsuda K. CDC20, a potential cancer therapeutic target, is negatively regulated by $\mathrm{p} 53$. Oncogene. 2008; 27:1562-1571.

29. Wang Z, Wan L, Zhong J, Inuzuka H, Liu P, Sarkar FH, Wei W. Cdc20: a potential novel therapeutic target for cancer treatment. Curr Pharm Des. 2013; 19:3210-3214.

30. Park EJ, Lim JH, Nam SI, Park JW, Kwon TK. Rottlerin induces heme oxygenase-1 (HO-1) up-regulation through reactive oxygen species (ROS) dependent and PKC deltaindependent pathway in human colon cancer HT29 cells. Biochimie. 2010; 92:110-115.

31. Valacchi G, Pecorelli A, Sticozzi C, Torricelli C, Muscettola M, Aldinucci C, Maioli E. Rottlerin exhibits antiangiogenic effects in vitro. Chem Biol Drug Des. 2011; 77:460-470.

32. Park EJ, Kwon TK. Rottlerin enhances IL-1beta-induced COX-2 expression through sustained p38 MAPK activation in MDA-MB-231 human breast cancer cells. Exp Mol Med. 2011; 43:669-675.

33. Singh BN, Kumar D, Shankar S, Srivastava RK. Rottlerin induces autophagy which leads to apoptotic cell death through inhibition of PI3K/Akt/mTOR pathway in human pancreatic cancer stem cells. Biochem Pharmacol. 2012; 84:1154-1163.

34. Kumar D, Shankar S, Srivastava RK. Rottlerin induces autophagy and apoptosis in prostate cancer stem cells via PI3K/Akt/mTOR signaling pathway. Cancer Lett. 2014; 343:179-189.

35. Lu W, Lin C, Li Y. Rottlerin induces Wnt co-receptor LRP6 degradation and suppresses both Wnt/beta-catenin and mTORC1 signaling in prostate and breast cancer cells. Cell Signal. 2014; 26:1303-1309.

36. Huang M, Tang SN, Upadhyay G, Marsh JL, Jackman CP, Srivastava RK, Shankar S. Rottlerin suppresses growth of human pancreatic tumors in nude mice, and pancreatic cancer cells isolated from $\mathrm{Kras}(\mathrm{G} 12 \mathrm{D})$ mice. Cancer Lett. 2014; 353:32-40.

37. Daveri E, Valacchi G, Romagnoli R, Maellaro E, Maioli E. Antiproliferative Effect of Rottlerin on Sk-Mel-28 Melanoma Cells. Evidence-based complementary and alternative medicine. 2015; 2015:545838.

38. Xie Q, Wu Q, Mack SC, Yang K, Kim L, Hubert CG, Flavahan WA, Chu C, Bao S, Rich JN. CDC20 maintains tumor initiating cells. Oncotarget. 2015; 6:13241-13254. doi: 10.18632/oncotarget.3676.

39. Mao DD, Gujar AD, Mahlokozera T, Chen I, Pan Y, Luo J, Brost T, Thompson EA, Turski A, Leuthardt EC, Dunn GP, Chicoine MR, Rich KM, et al. A CDC20-APC/SOX2 Signaling Axis Regulates Human Glioblastoma Stem-like Cells. Cell Rep. 2015; 11:1809-1821.

40. Ji P, Zhou X, Liu Q, Fuller GN, Phillips LM, Zhang W. Driver or passenger effects of augmented c-Myc and Cdc20 in gliomagenesis. Oncotarget. 2016. doi: 10.18632/ oncotarget.8080.

41. Liao YF, Hung YC, Chang WH, Tsay GJ, Hour TC, Hung HC, Liu GY. The PKC delta inhibitor, rottlerin, induces apoptosis of haematopoietic cell lines through mitochondrial membrane depolarization and caspases' cascade. Life Sci. 2005; 77:707-719.

42. Ringshausen I, Oelsner M, Weick K, Bogner C, Peschel C, Decker T. Mechanisms of apoptosis-induction by rottlerin: therapeutic implications for B-CLL. Leukemia. 2006; 20:514-520.

43. Valacchi G, Pecorelli A, Sticozzi C, Torricelli C, Muscettola M, Aldinucci C, Maioli E. Rottlerin exhibits antiangiogenic effects in vitro. Chem Biol Drug Des. 2011; 77:460-470.

44. Huang M, Tang SN, Upadhyay G, Marsh JL, Jackman CP, Srivastava RK, Shankar S. Rottlerin suppresses growth of human pancreatic tumors in nude mice, and pancreatic cancer cells isolated from $\mathrm{Kras}(\mathrm{G} 12 \mathrm{D})$ mice. Cancer Lett. 2014; 353:32-40.

45. Zeng X, Sigoillot F, Gaur S, Choi S, Pfaff KL, Oh DC, Hathaway N, Dimova N, Cuny GD, King RW. Pharmacologic inhibition of the anaphase-promoting complex induces a spindle checkpoint-dependent mitotic arrest in the absence of spindle damage. Cancer Cell. 2010; 18:382-395.

46. Sackton KL, Dimova N, Zeng X, Tian W, Zhang M, Sackton TB, Meaders J, Pfaff KL, Sigoillot F, Yu H, Luo X, King RW. Synergistic blockade of mitotic exit by two chemical inhibitors of the APC/C. Nature. 2014; 514:646-649.

47. Zhang L, Niu T, Huang Y, Zhu H, Zhong W, Lin J, Zhang Y. Compound 331 selectively induces glioma cell death by upregulating miR-494 and downregulating CDC20. Sci Rep. 2015; 5:12003.

48. Jiang J, Thyagarajan-Sahu A, Krchnak V, Jedinak A, Sandusky GE, Sliva D. NAHA, a novel hydroxamic acidderivative, inhibits growth and angiogenesis of breast cancer in vitro and in vivo. PLoS One. 2012; 7 :e34283. 
49. Das T, Roy KS, Chakrabarti T, Mukhopadhyay S, Roychoudhury S. Withaferin A modulates the Spindle assembly checkpoint by degradation of Mad2-Cdc20 complex in colorectal cancer cell lines. Biochem Pharmacol. 2014; 91:31-39.

50. Jiang J, Jedinak A, Sliva D. Ganodermanontriol (GDNT) exerts its effect on growth and invasiveness of breast cancer cells through the down-regulation of CDC20 and uPA. Biochem Biophys Res Commun. 2011; 415:325-329.

51. Regenbrecht CR, Jung M, Lehrach H, Adjaye J. The molecular basis of genistein-induced mitotic arrest and exit of self-renewal in embryonal carcinoma and primary cancer cell lines. BMC Med Genomics. 2008; 1:49.
52. Zhang L, Yang B, Zhou K, Li H, Li D, Gao H, Zhang T, Wei D, Li Z, Diao Y. Potential therapeutic mechanism of genistein in breast cancer involves inhibition of cell cycle regulation. Mol Med Rep. 2015; 11:1820-1826.

53. Wang L, Ye X, Cai X, Su J, Ma R, Yin X, Zhou X, Li H, Wang Z. Curcumin suppresses cell growth and invasion and induces apoptosis by down-regulation of Skp2 pathway in glioma cells. Oncotarget. 2015. doi: 10.18632/ oncotarget.4090. 